\title{
LA ECONOMÍA POLÍTICA EMPÍRICAMENTE FUNDAMENTADA E HISTÓRICAMENTE CONTEXTUALIZADA DE CELSO FURTADO
}

\author{
James M. Cypher*
}

RESUMENः Celso Furtado, creador de la economía política estructuralista latinoamericana, llamó la atención del mundo con la construcción de un proyecto factible de desarrollo nacional para Brasil. Sofisticado defensor del cambio estructural, Furtado representó a un reformismo radical y de vanguardia basado en el análisis pragmático del subdesarrollo, «la» condición subyacente de las naciones periféricas. El objetivo de este artículo es ofrecer tanto una síntesis como una evaluación de sus contribuciones al campo de la economía política como parte de la teoría del desarrollo. La hipótesis central de este artículo plantea que la postura metodológica y analítica de Furtado - en particular el enfoque dinámico e históricamente contextualizado y la tendencia a centrar el desarrollo en la capacidad tecnológicamerece más amplia aceptación y mayor aclamación. Una hipótesis adicional sostiene que, si bien el trabajo de Furtado se asemeja al trabajo del institucionalismo radical estadounidense en su etapa inicial (particularmente el trabajo de Veblen), tanto Furtado como sus seguidores perdieron la importante oportunidad de explorar complementariedades y sinergias que quizá hubieran forjado una perspectiva más robusta para renovar el desarrollismo furtadiano en la actualidad a lo largo de América Latina.

pala bras Clave Celso Furtado, desarrollismo, estructuralismo, dependencia, especificidad histórica.

\footnotetext{
* Profesor-investigador del Doctorado en Estudios del Desarrollo de la Universidad Autónoma de Zacatecas, México.

«The Origins of Developmentalist Theory: The Empirically-Based, Historically-Contextualized Political Economy of Furtado», International Journal of Political Economy (2015), here translated and published by permission of Taylor y Francis, LLC. http://www.tandfonline.com

Traducción de Cynthia Arredondo Cabrerra
} 
ABSTRACT: Celso Furtado, a creator of Latin American Structuralist Political Economy, was riveted upon the construction of a viable national development project for Brazil. As a sophisticated advocate for structural change, he represented forward-looking reformism based in a pragmatic analysis of underdevelopment, «the» underlying condition of peripheral nations. The objective of this article is to offer both a synthesis and an evaluation of his contributions to the political economy of development economics. The hypothesis of this article is that Furtado's methodological/analytical stance - in particular, his dynamic, historically-contextualized, approach and his tendency to center development on technological capacity - merits broader acceptance and greater acclaim. An ancillary hypothesis maintains that, while Furtado's work paralleled that of early US institutionalism (particularly that of Veblen), he and his followers have thus far missed an important opportunity to explore the complementarities and synergies that might have been forged to renovate the Furtadian developmentalist perspective.

KEY WORDS: Celso Furtado, developmentalism, structuralism, dependence, historical specificity. 


\section{INTRODUCCIÓN}

elso Furtado (1920-2004) ha sido, en todos los sentidos, el econo-
mista más aclamado de Brasil. Su larga carrera profesional fue des-
tacada en casi todos los aspectos. A temprana edad su investigación ya había logrado ser ampliamente distribuida en el mundo angloparlante. ${ }^{1}$ Furtado tuvo la gran fortuna de estar situado en el lugar preciso para convertirse en el creador y desarrollador de la economía política estructuralista latinoamericana al haber sido seleccionado por Raúl Prebisch para liderar la División de Desarrollo Económico (1950-1957) en la recién formada Comisión Económica para América Latina y el Caribe (CEPAL) con sede en Chile. Furtado se integró a la CEPAL en 1949, luego de haber terminado su tesis de doctorado en la Sorbona en 1948. Después de haber experimentado un compañerismo muy cercano al ser miembro del grupo de economistas más innovador e influyente de Latinoamérica - contando además con la tutoría de Prebisch — , Furtado se instaló en Cambridge, Inglaterra, donde por un año trabajó con N. Kaldor y otros eminentes keynesianos. Fue durante este periodo que termina su libro más citado, impreso en 1959 y subsecuentemente traducido al inglés y publicado como The Economic Growth of Brazil (Furtado, 1971). Este trabajo se ha mantenido, incluso en el siglo Xxi, como un hito del análisis económico (De Araújo, Macambira y Teixeira, 2009).

En 1958 regresó a Brasil para trabajar, hasta 1964, como asesor económico nacional y formulador de políticas. Justo antes de su exilio en 1964 - resultado de un golpe de Estado militar respaldado en su totalidad por el gobierno de Estados Unidos - la búsqueda de Furtado de políticas desarrollistas factibles para el paupérrimo noreste de Brasil, lo impulsaron a abogar abiertamente por una reforma agraria. Esta situación lo llevó a enfrentar el primer revés de su carrera: en 1963 fue

${ }^{1}$ El ensayo de Furtado «Capital Formation and Economic Development» («Formación de capital y desarrollo económico») fue publicado en Brasil en 1951; subsecuentemente, esta publicación apareció en inglés en International Economic Papers, núm. 4, en colecciones de literatura de más amplia distribución sobre la economía del desarrollo (Agarwala y Singh, 1958). 
cesado de su posición como ministro de Planeación de Brasil. En el exilio Furtado inició una nueva carrera como profesor en la Universidad de París I (1965-1980). Durante este periodo dejó de centrar su atención en la construcción de un proyecto factible de desarrollo nacional. Como era de esperarse, en lugar de ello su enfoque analítico cambió drásticamente otorgando mayor relevancia al papel del capital transnacional en la desnacionalización de la base productiva; un proceso que llevó a la creación de estructuras de dependencia nacional en los países periféricos. Para 1979, Furtado pudo regresar a Brasil, donde ocupó una variedad de posiciones como funcionario público experto y como formulador de políticas públicas hasta su muerte en 2004. Fue durante este periodo cuando Furtado, maduro a base de pruebas de fuego, renovó su enfoque, ahora esencialmente optimista, sobre las potencialidades de lograr a través de la construcción creativa un proyecto factible de desarrollo nacional. Es importante señalar que la bien pensada postura de Furtado fue criticada por muchos, como F. H. Cardoso - presidente de Brasil de 1995 a 2002 y creador de la teoría del «desarrollo dependiente asociado»-, quien argumentó que «la confianza de Furtado en la capacidad del Estado para planear y liderar un proceso endógeno de desarrollo autosuficiente era exagerado» (Cardoso, 2005: 5).

En el intento de analizar la vasta carrera de Furtado, es importante comprender que fue un líder que se infiltró muy sofisticadamente en las estructuras gubernamentales para avanzar de manera exitosa en el impulso de un cambio estructural. Él representó un reformismo de vanguardia basado en el análisis pragmático del subdesarrollo como la condición subyacente de las naciones periféricas (generada siglos atrás a través del ejercicio del poder colonial). Esta condición, sostenía Furtado, no constituía una etapa y podía ser trascendida a través de la planeación de proyectos, la capacidad del Estado y una reconstrucción institucional.

A lo largo de su vida, Furtado publicó de manera regular un gran número de artículos y libros — una exhaustiva obra que ha sido recientemente reanalizada - principalmente en Brasil (véase, por ejemplo, Boianovsky, 2008; Corsi y Camargo, 2010; Guillén y Vidal, 2008; 
Mallorquin, 2005; Tavares, 2000). Sin embargo, fuera de Brasil y en general en Latinoamérica, el aporte de Furtado al desarrollo ha sido poco reconocido: como muestra se omitió cualquier referencia a Furtado en la primera edición de The New Palgrave: Economic Development, su nombre fue mencionado apenas una vez en The Origins of Development Economics e increíblemente fue excluido de Fifty Key Thinkers on Development (Eatwell, Milgate y Newman, 1989; Jomo y Reinart, 2005; Simon, 2006).

No es el objetivo de este artículo presentar una disección detallada de las obras de Furtado, tampoco el hacer una exploración en «la historia del pensamiento». Más bien este artículo ofrece tanto una síntesis como una evaluación de sus contribuciones a la economía política dentro de la economía del desarrollo. Este artículo no es un ejercicio exegético de la historiografía de las ideas; es un esfuerzo para identificar las aportaciones de Furtado a la economía política en 1) el estructuralismo, 2) la contextualización histórica, y 3) la capacidad tecnológica, lo cual podría facilitar la reformulación de una política de desarrollo posneoliberal. La hipótesis de este artículo sostiene que la postura metodológica-analítica de Furtado - en particular su enfoque dinámico de contextualización histórica, así como su tendencia a centrar el desarrollo en la capacidad tecnológicamerece más amplia aceptación y mayor aclamación. Una hipótesis adicional sostiene que, si bien el trabajo de Furtado se asemeja al trabajo del institucionalismo estadounidense radical en su etapa inicial (particularmente el trabajo de Veblen), tanto Furtado como sus seguidores perdieron la importante oportunidad de explorar complementariedades y sinergias que quizá hubieran forjado una perspectiva renovada del desarrollismo furtadiano; problemática analizada en la última sección de este artículo.

\section{SITUANDO A FURTADO: ¿QUÉ ES LA "CIENCIA" ECONÓMICA?}

Por lo menos en Brasil ha habido intentos acríticos de delimitar la «economía» en tres distintas áreas - microeconomía, macroeconomía y economía del desarrollo - sin tomar en cuenta el hecho de que, en buena medida, tal 
intento de delimitación ensombrece las mutuas tensiones y las incongruencias contenidas en esta triple división (véase Bresser-Pereira, 2007: 14-17). Por ejemplo, como es cada vez más evidente, la microeconomía neoclásica ha establecido una prolongada guerra contra la existencia de cualquier intento de demarcar la macroeconomía como un campo de análisis independiente a través de la campaña de «micro-fundamentos de la macroeconomía». ${ }^{2}$ Mientras tanto, la economía del desarrollo ha sido sumariamente descartada como un campo de estudio separado, particularmente por uno de los más célebres macroeconomistas —un individuo sin absolutamente ningún tipo de credenciales (o análisis) que ofrecer, lo que podría haber legitimado su intervención- -3 No obstante, este ataque ha resonado dentro, y aún más allá de la propia economía del desarrollo. Aparentemente, fue suficiente que un aclamado economista del campo del comercio exterior («validado» al recibir el Premio en Ciencias Económicas del Banco de Suecia, también conocido popularmente como Premio Nobel de Economía) haya acusado a los principales economistas del desarrollo de ofrecer no más que meras presentaciones retóricas, creando de ese modo una «nueva escuela del desarrollo [basada] en metáforas sugestivas, realismo institucional (sic), razonamiento interdisciplinario y una actitud relajada hacia la consistencia interna ${ }_{+}{ }^{4}$ De acuerdo con esta autoproclamada «autoridad», «la teoría del desarrollo fue abandonada» porque «las investigaciones clásicas sobre el campo comenzaron a parecer [... ] simplemente incomprensibles» (Krugman, 1995: 23). Esta misma «autoridad» afirmó, arrogantemente, que «la economía del desarrollo era

\footnotetext{
${ }^{2}$ Para un análisis de su trayectoria enfocada a la economía del desarrollo, véase Herrera (2006); para un crítica amplia y devastadora de su trayectoria, ahora ligada al surgimiento de la hegemonía neoliberal en un análisis económico ortodoxo, véase Mirowski (2013).

${ }^{3}$ Véase Krugman (1995), «The Fall and Rise of Development Economics», op. cit. Este capítulo es asignado ampliamente a los estudiantes de nivel licenciatura en las «mejores» universidades de Estados Unidos.

${ }^{4}$ En el texto original de la conferencia de Krugman sobre el modelo de Ohlin, presentada en la Stockholm School of Economics en el otoño de 1992, incluye esta cita y una serie de burlas similares que fueron eliminadas de la versión publicada en 1995. El texto original de la conferencia puede consultarse en http://web.mit.edu/krugman/www/dishpan.html
} 
de estilo arcaico, aún para su propio tiempo» (Krugman, 1995: 24). El elemento que reforzó su diatriba fue la revelación de una imperdonable naturaleza transgresora de los «pioneros del desarrollo» (incluyendo a Albert Hirschman, Arthur Lewis, Gunnar Myrdal y Paul RosensteinRodan), pues, según dijo el «experto» autoproclamado, fracasaron en la construcción de modelos. Esto colocó todos los esfuerzos de los economistas del desarrollo afuera de los límites de la «economía», dado que «la teoría económica es esencialmente una colección de modelos» (Krugman, 1995: 27). Irónicamente, la fecha de publicación de estas «observaciones» coincidió con la publicación de la crítica a la economía ortodoxa de Heilbroner y Milberg, la cual resaltó la «extraordinaria indiferencia» de los profesionales dentro del paradigma dominante, quienes no tienen absolutamente ninguna preocupación por encontrar las conexiones entre su manía de construcción de modelos teóricos y la realidad (Heilbroner y Milberg, 1995: 3-4). Al respecto, estos autores encontraron evidencia de una profunda espiral analítica descendente que había dejado fuera a la corriente predominante de los llamados «mejores» economistas neoclásicos (como Krugman) de las corrientes históricas que han definido la economía política como un campo de estudio (como Smith, Ricardo, Marx, Mill, etcétera). La economía neoclásica contemporánea, argumentaron, ha clasificado todas las cuestiones que le resultan imposibles de analizar dentro del marco de sus modelos matemáticos como «problemas no económicos» (Heilbroner y Milberg, 1995: 7).

Las tendencias apoyadas por Truman, y las que Heilbroner y Milberg deploraron, ganaron ímpetu hacia mediados de la década de 1990 convergiendo con la creación y propagación del modelo del equilibrio general dinámico estocástico (EGDE), el cual se convirtió en el enfoque dominante de todo análisis macroeconómico en las universidades de élite, incluyendo la teoría del crecimiento (que, según los neoclásicos abarca el «desarrollo»).

Aquí se presenta una descripción reciente del modelo EGDE ofrecida por el Comité de Ciencia y Tecnología de la Cámara de Representantes de Estados Unidos: 
El término «general» abarca [...] todos los mercados en la economía. «Equilibrio» [significa] un balance entre oferta y demanda $[. .$.$] y la competencia reinan$ en los mercados sin ser la existencia de la escasez [...] o el desempleo [...] «Estocástico» corresponde a [...] la aleatoriedad manejable [...] que consiste en eventos inesperados $[\ldots]$ pero asume que los agentes del modelo pueden asignar una probabilidad matemática correcta a [todos] esos eventos.

Los agentes [...] son dotados de [...] clarividencia. Inmortales, ven el final de los tiempos y son conscientes de todo lo que posiblemente pudiera ocurrir [...]; sus decisiones son siempre instantáneas y nunca incorrectas [...] todos [...] los individuos o empresas tienen necesidades y gustos idénticos [...] que como "optimizadores» persiguen con ilimitado interés individual y pleno conocimiento [...] (citado por Mirowski, 2013: 276, cursivas añadidas).

Ante las estrepitosas críticas hacia este modelo y su enfoque, de reciente formulación, junto con el inicio de la Gran Recesión a finales de 2007, los practicantes de la ortodoxia han buscado evadir e ignorar estas críticas aferrándose, cada vez más, a su consagrado enfoque del EGDE (Mirowksi, 2013: 276-294).

Con el cambio de milenio, el enfoque de Furtado al desarrollo (y cualquier otro enfoque que no derive de los supuestos del modelo EGDE) fue enteramente adjudicado al rubro de los temas «no económicos», asimismo declarado como «incomprensible». No obstante, una modesta contracorriente conocida algunas veces como «heterodoxia» - que incluye los enfoques del institucionalismo vebleniano y del «neo-schumpeterianismo» sobre el tema del desarrollo, así como el análisis económico «evolutivo» - ha persistido en cierta medida. Es en esta contracorriente que encontramos a aquellos que trabajan en el tema de la teoría económica del desarrollo; aquellos que no se circunscriben en el cada vez más esperpéntico discurso neoclásico. Sobre todo, en Brasil, particularmente desde finales de 2002, cuando el poder del Estado se desplazó hacia las fuerzas progresistas nacionalistas, una postura heterodoxa emergente en la política pública fomentó el resurgimiento del desarrollismo furtadiano, denominado como «nuevo» o «neodesarrollismo» (Bresser-Pereira, 2011; Cypher, 2012). 


\section{EL ESTRUCTURALISMO DE FURTADO}

Furtado se doctoró en la Sorbona bajo la guía de destacados estructuralistas franceses e historiadores económicos, incluyendo especialmente a F. Perroux:

Perroux (1939) definió la economía estructural como la ciencia de las relaciones que caracterizan un sistema económico situado en determinados tiempo y espacio. Central para el enfoque de Perroux era la visión de que por encima de los «elementos que se dan por sentados» de la teoría neoclásica (preferencias, recursos y tecnología), el análisis de las instituciones y las estructuras en el tiempo tendrían que situarse en el centro mismo del análisis económico. Una importante e innovadora contribución de Perroux apunta a su teoría de la dominación, que es central en la construcción conceptual [de la CEPAL] de un sistema económico: en lugar de estar constituido por relaciones entre [entidades] iguales, el mundo económico se conceptualiza a partir de relaciones ocultas o explícitas de «fuerza», «poder» $y$ «limitaciones sociales» entre entidades dominantes y entidades dominadas (Blankenburg, Palma y Tregenna, 2008: 3).

No obstante, Furtado señaló que el estructuralismo de la CEPAL no se basaba directamente en Perroux; más bien, este método se fundaba parcialmente en un análisis dinámico de lo que era - y es - considerado como "parámetros no económicos en modelos macroeconómicos», tal como la estructura agraria, el atraso tecnológico, el desempleo, «el control de las empresas, la composición de la fuerza laboral, etc.» (Furtado, 1987: 209-10). La problemática más profunda tiene que ver con quién ocupa la posición hegemónica ideológica para delimitar el campo de estudio y así, de manera arbitraria y apriorística, considerar como temas «no económicos» todos aquellos que no son fáciles de incorporar dentro de los angostos límites de los modelos económicos basados en supuestos de una nación desarrollada sujeta a condiciones de competitividad perfecta impulsada por tendencias armoniosas, estables, de equilibrio óptimo de los mercados y con condiciones de «información perfecta» del individualismo metodológico. 
Debido a su educación en Francia y al rápido ascenso en la CEPAL, Furtado desarrolló sus ideas estructuralistas medulares en estos dos únicos ambientes, no contaminados por las condiciones de la espiral descendente que arrasó la rama de las ciencias económicas en las décadas de 1930 y 1940. En este descenso se encuentra la participación de L. Robbins, quien buscó, siendo director de la influyente London School of Economics en 1929, redefinir «el campo de estudio» de la «ciencia económica». En 1932, Robbins publicó la primera edición de An Essay on the Nature and Significance of Economic Science (Ensayo sobre la naturaleza e importancia de la ciencia económica), cuyo objetivo era reescribir la bistoria para obliterar el reconocimiento que A. Marshall había otorgado a los métodos y análisis de la escuela histórica alemana (Hodgson, 2001: 95-112). Robbins acuñó el popular argumento de que la «ciencia económica» podría estar interesada únicamente en la asignación de recursos escasos (bajo condiciones de pleno empleo sin capacidad ociosa) para determinados fines. Esta «definición» de los límites del análisis pretendía aplicarse a todo régimen socioeconómico sin importar el tiempo o lugar en que éstos se ubicasen. «Robbins vio la economía como la exploración a priori de las deducciones de los axiomas de la elección racional» (Hodgson, 2001: 209). «La economía ya no tendría un campo institucional o histórico específico de análisis» (Hodgson, 2001: 207).

Robbins buscó, y eventualmente alcanzó la hegemonía intelectual sobre los «teóricos generales» quienes no «[...] reconocen el problema de la especificidad histórica porque creen que la economía puede enteramente proceder sobre la base de supuestos universales e históricamente indefinidos» (Hodgson, 2001: 28). Posteriormente, en 1974 Robbins ayudó a formar la influyente Sociedad Mont Pèlerin, estableciendo ahí los fundamentos de una doctrina que sería después conocida como neoliberalismo (Mirowski, 2013: 6-8). Su ensayo formativo de 1932 creó una inercia gigantesca que rápidamente deslegitimó cualquier forma de análisis económico, salvándose los ejercicios deductivos más obtusos y estériles cobijados por un rígido neoclasicismo y la teoría de la escuela austriaca. Parte de esta deslegitimación se debió a la disposición de J. 
Schumpeter de dirigir acusaciones sumamente injuriosas (incluyendo incompetencia) contra la escuela histórica alemana y Veblen (Schumpeter, 1930). Poco después, en 1947, P. Samuelson facilitó la consolidación de la hegemonía ideológica de los planteamientos de Robbins, pues su texto introductorio marcó una nueva época al definir estrechamente los parámetros y límites de lo que los entendidos en el tema pudieran analizar en la rama de la llamada «ciencia» económica, dejándolos en un mundo estático y ahistórico.

En el mismo momento en que la campaña de Robbins, Schumpeter y Samuelson abrumó a los defensores de la economía política, la escuela estructuralista latinoamericana, en su estado embrionario - inmersa en su entorno distinto y distante-, se materializó rápidamente. La "problemática» del «desarrollo» fue percibida como un nuevo campo que demandaba un método separado de análisis, como Furtado repetidamente señaló. Tres son las situaciones que se pueden distinguir en el avance del estructuralismo latinoamericano, a saber: 1) el relativo aislamiento de la CEPAL de la metodología predominante que impactó ampliamente la ciencia económica anglosajona; 2) la condición de independencia institucional al ser el primer centro de investigación de la Organización de las Naciones Unidas (onu) que se estableció en el Sur global, y 3) la irrelevancia de la corriente económica predominante para el análisis de estructuras económicas heterodoxas - reconocidas incluso por muchos de los economistas de la corriente predominante, quienes ayudaron a impulsar la economía del desarrollo después de la Segunda Guerra Mundial-, circunstancias que permitieron a Furtado y sus colegas establecidos en Santiago de Chile avanzar sin mayores impedimentos (momentáneamente) en su propia investigación fundada en explicaciones estructuralistas.

\section{¿DESARROLLO AUTÉNTICO O "DEPENDENCIA»?}

Dado que la matriz para la determinación y análisis del «condicionamiento» de fuerzas y factores puede ser dividida (esquemáticamente) en elementos 
endógenos/exógenos, los economistas de la CEPAL, en particular Furtado durante su exilio, presentaron su versión «estructuralista» de la teoría de la «dependencia». Esta versión, aunque superficialmente similar, se diferenciaba bastante de la versión propuesta por A. G. Frank, así como de las de los seguidores de la teoría del sistema mundo (Blankenburg, Palma y Tregenna, 2008; Cypher, 1979; Palma, 1978). El objetivo del trabajo de la CEPAL no era abandonar el estudio de la formación nacional del subdesarrollo, tampoco negar la contribución de los países subdesarrollados a las causas primordiales de su condición; más bien el objetivo era aportar un «enfoque integral [...] para incrementar nuestro conocimiento acerca de los vínculos entre las relaciones externas y las formas internas de la dominancia social» (Furtado, 1987: 210). En la búsqueda de diversos aspectos de la «dependencia estructural», nuevas fuerzas y factores - incluyendo el Estado y las firmas transnacionales - fueron puestos en el primer plano del análisis. No obstante, los economistas de la CEPAL teorizaron inadecuadamente el Estado, desaprovechando la oportunidad que tuvieron de hacerlo (Alavi, 1982; Cypher, 1990).

El nombre de Furtado ha sido constantemente asociado con la teoría de la «dependencia», sin embargo el enfoque de Furtado sobre el desarrollo no tenía nada en común con el muy conocido argumento del pensamiento desarrollista que señala que el desarrollo autónomo era imposible. Furtado argumentó que «una reestructuración para superar el subdesarrollo se basa más en un ordenamiento integral que en el ordenamiento proporcionado por los mercados, y tal ordenamiento sólo es posible a través de la planificación» (Furtado, 1987:225 cursivas añadidas). Esta planificación debería incluir la diversificación de las exportaciones, como la que se había logrado en Japón a través de su Ministerio de Comercio Internacional e Industria (Furtado, 1987: 224). En rechazo a la «ortodoxia marxista», la planificación sería parcial y limitada, pues solamente «ciertas decisiones pueden ser centralizadas de manera favorable», como la dispersión geográfica de la base industrial o «el incremento de la importancia relativa de las industrias de bienes de capital» (Furtado, 1970: 193-194). En resumen, la «dependencia» externa es un elemento 
dado de la estructura del subdesarrollo y no necesariamente consigna a las naciones a un estado de perpetua degradación, particularmente porque el Estado tiene la capacidad potencial de emplear los excedentes económicos en la promoción de políticas de redistribución del ingreso y «desarrollo auténtico» (véase recuadro 1) a través de un proceso constructivo de «"desestructuración" social» (Furtado, 1987: 224-225).

\section{Recuadro 1}

Pre-requisitos para un «Desarrollo Auténtico»

- Con el objetivo de alcanzar un elevado índice de desarrollo, conforme a criterios verdaderamente sociales, tendremos que llevar a cabo algunos cambios importantes en nuestras estructuras básicas.

- [Debemos] crear las condiciones para un cambio rápido y eficaz en la estructura agraria arcaica del país.

- Tenemos que andar intrépidamente el camino del cambio constitucional que permitirá una reforma agraria y un cambio radical de la administración gubernamental del sistema fiscal y de la estructura bancaria.

- Debemos subordinar la acción de Estado a una definición clara de los objetivos del desarrollo económico y social.

- Debemos tener estatutos legales para subordinar la acción del capital extranjero a los objetivos del desarrollo económico y a los requerimientos de una independencia política.

- Y sobre todo, todos debemos tener un plan para el desarrollo económico y social compatible con nuestras posibilidades y en conformidad con las aspiraciones de la gente.

Fuente: Furtado, 1963, pp. 534-535.

El enfoque estructuralista presentado por Furtado tendió a dar prioridad a la formación nacional, no obstante este enfoque requería una comprensión de América Latina y sus «realidades sociales que presentan peculiaridades y cuyas características deben ser conocidas para entender 
el comportamiento del sistema económico» (Furtado, 1970: 72). Dado que las formas de la estructura social de América Latina tienen su origen en la colonización, las estructuras económicas se construyeron en cierta medida en relación con las demandas impuestas y oportunidades creadas por la producción y el comercio coloniales. En paralelo con los operadores del comercio internacional basados en las grandes ciudades, impulsado desde el exterior, Furtado enfatizó los papeles autónomos de la élite terrateniente y del aparato estatal ascendente: "Emergió una estructura de poder tripartita donde los factores "externos/internos" se entremezclan, dando luz a una matriz social simultáneamente determinada, así acallando las tensiones, supuestamente dicotómicas, de lo "externo/interno"» (Furtado, 1970: 71-109).

Eventualmente, Furtado condensó su pensamiento sobre el desarrollo en dos modelos estructurales. El primero fue el caso de «estructura simple del subdesarrollo» o la economía bibrida (condición A en la figura 1), el cual tuvo la capacidad de evolucionar hacia una estructura de tres sectores denominada «etapa avanzada del subdesarrollo» (condición B en la figura 1). Al hacer una distinción clara entre la estructura (forma) y el proceso (causalidad), Furtado reprendió a los estructuralistas que no habían enfatizado la importancia del papel de los factores cualitativos: «Al confinarse al plano de la descripción morfológica y excluyendo la noción de causalidad, el enfoque estructural reduce el horizonte cognitivo» (Furtado, 1983: 182, cursivas añadidas). La creatividad bumana puede ser aplicada para conducir capacidades de innovación que trasciendan los obstáculos del subdesarrollo (como el modelo de la figura 1), abriendo la senda al «desarrollo auténtico».

La figura 1 presenta algunos detalles respecto a la frecuentemente citada definición de Furtado de desarrollo como «un proceso especial debido a la penetración de modernas compañias capitalistas en estructuras arcaicas» (Furtado, 1964: 138, cursivas añadidas).

A lo largo del tiempo, Furtado osciló entre dos perspectivas de la trayectoria histórica de su modelo de dos estructuras. A veces se adhería a una perspectiva de estancamiento, pobremente articulada, de la estructura 


\section{FIGURA 1}

El modelo de subdesarrollo de dos estructuras de Furtado

A. 'Estructura subdesarrollada simple' : Economía Híbrida

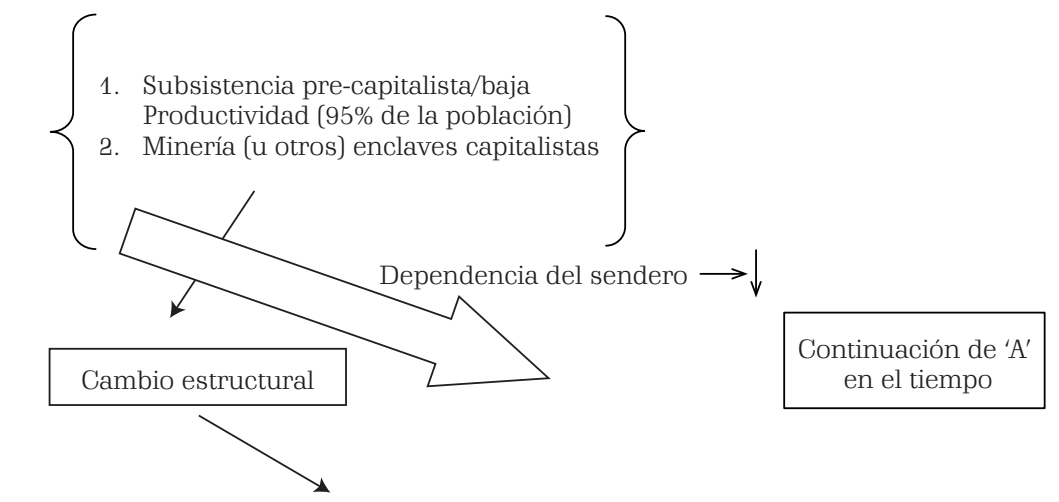

B. Etapa Avanzada del Subdesarrollo: Economía de Tres Sectores

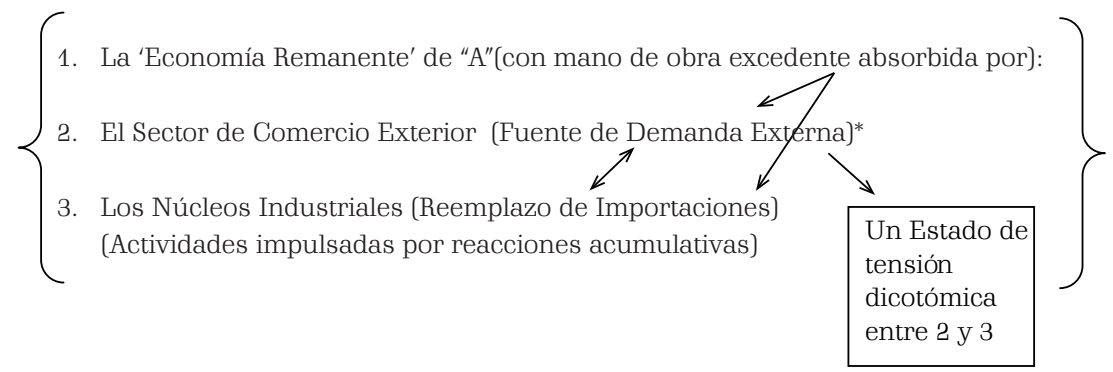

Fuente: Descripción y resumen propio del autor basándose en Furtado (1964: 129-138).

* Implicando efectos multiplicadores internos.

de tres sectores en la «etapa avanzada de subdesarrollo», y en otras ocasiones se encontraba más orientado a la postura de «reformador progresista» sosteniendo que a través de un Estado activista y una planificación estratégica (lo que sin duda hoy podría denominarse como la "política industrial») la estructura en la «etapa avanzada» podría encontrar un camino para adelantar en el desarrollo autónomo (Furtado, 1963; 1964: 129-138; 1970: 83-108; 1987). En cualquier caso, Furtado no señaló que necesariamente ha de haber un movimiento teleológico entre $A$ y $B$. 
El análisis de la condición «A» fue, sin duda, el más grande logro de Furtado, cuya mayor expresión apareció en The Economic Growth of Brazil (Furtado, 1971). El libro ejemplificó la contextualización y la especificidad históricas que pusieron de manifiesto la observación clave de Sombart: "No hay economía en abstracto, sino una vida económica particularmente constituida e históricamente distinguible» (Sombart, 1930: 196). Asimismo, Furtado presenta en su libro un profundo análisis de la estructura tripartita del poder arriba mencionada, con especial énfasis en la estructura de la tenencia de la tierra y la generación de excedentes de la pequeña y antediluviana élite que poseía virtualmente toda la tierra. Como ha sido señalado en la figura 1, en este «modelo» sólo alrededor de 5 por ciento de la fuerza laboral es empleada por los sectores minero y agrario; son estos sectores los que tienen amplio dominio sobre la colonia y reciben desmesurados excedentes económicos. Siguiendo los dictados de la ventaja comparativa, la economía hibrida languidece en las trampas de la dependencia de los recursos naturales: bajos ingresos y baja productividad. Esta circunstancia no es una fase histórica, es una condición estructural.

No obstante, las naciones no han de sentirse condenadas. Consciente de la hipótesis de Prebisch-Singer, Furtado argumentó que la tendencia a la baja de los precios de las materias primas no constituía necesariamente un destino. La proto-industrialización - cuando es acompañada por políticas apropiadas del Estado- podría ser la respuesta en momentos históricos bajo las siguientes condiciones: 1) precios de las materias primas altos; 2) un proceso dinámico de diversificación de las exportaciones, y 3) nuevos sistemas de tenencia de la tierra, menos concentrados. Estos pasos incipientes hacia un cambio estructural fueron posibles en tanto comerciantes, terratenientes y empresas financieras comenzaron un proceso espontáneo de sustitución de importaciones fortalecido a través de políticas estatales viables; tal fue el caso de Brasil en los años previos a la Gran Depresión (Font, 2010; Topik, 1987).

En Brasil, el proceso de cambio estructural eventualmente se consolidó debido a la presión causada por la Gran Depresión para reenfocar, 
casi por completo, sus políticas hacia el crecimiento del mercado interno. A través de una rápida expansión dirigida por el Estado del proceso de industrialización, desde la década de 1930 hasta finales de la dictadura militar en la década de 1980, emergió una nueva estructura de política económica, la que dio lugar al segundo modelo de Furtado: la Estructura de Tres Sectores denominada «Etapa Avanzada de Desarrollo»; en éste Furtado usó el modelo de Lewis de mano de obra excedente, el cual argumentaba que la subsistencia del sector pre-capitalista proporcionó una mano de obra vasta y barata que podría ser empleada para la expansión de los sectores comercial e industrial. Para ese entonces, el sector comercial ya era sustancialmente distinto del de la «economía colonial», pues las ganancias extraordinarias de este sector eran, al menos parcialmente, reinvertidas en diversas actividades económicas, que incluían tanto actividades emergentes relacionadas con el sector de comercio exterior, como manufactura para exportación, así como las relacionadas con la sustitución de importaciones o con los sectores industriales «estratégicos» apoyados a través de la promoción del Estado. Todo esto significó que el sector de comercio exterior se había convertido en una fuente dinámica que promocionaba el cambio interno, el cual fomentaba el crecimiento a través de efectos multiplicadores indirectos surgidos en respuesta a la reinversión de los excedentes en la economía nacional. En tanto la vasta mano de obra estaba siendo utilizada, el emergente sector industrial y el sector de comercio exterior en pleno proceso de expansión competirían por la mano de obra y por la hegemonía sobre la economía nacional. De este modo, un estado de "tensión dicotómica» entre los dos sectores más dinámicos definiría el nuevo modelo y, muy probablemente, ejercería fuerzas centrífugas con efectos aceleradores.

Furtado titubeó en relación con el peso explicativo que debía asignar a las fuerzas entrópicas y a las contradicciones endógenas dentro de su segundo modelo. Particularmente, después del golpe de Estado que lo mandó al exilio, Furtado adelantó su hipótesis, muy poco convincente, del estancamiento. Lo que hacía a la hipótesis poco creíble era el hecho de que la publicación de su obra difícilmente coincidía con los datos del 
periodo del «milagro brasileño» en el que el crecimiento promedio real anual del рів era de 10.1 por ciento de 1968 a 1976 (Baer, 2001: 462).

Furtado enfatizó que en el caso del modelo de Tres Sectores «no ha provocado ningún cambio en las condiciones de vida de tres cuartos de la población del país. La principal característica ha sido una creciente concentración, social y geográficamente, del ingreso» (Furtado, 1963: 527). Así continúa el argumento:

Éste ha proveído al país de los instrumentos para tomar decisiones, le ha otorgado la habilidad de ejercitar el poder de elección y, haciendo consciente a la gente de su destino, la ha hecho responsable de sus propios errores. La raíz causal del actual estado de descontento en Brasil es esta simple verdad: sabemos dónde yacen los errores de nuestro desarrollo, y sabemos que está en nuestro poder el erradicarlos o minimizarlos (Furtado, 1963: 527-528 cursivas añadidas).

\section{CIENCIA, TECNOLOGÍA Y DESARROLLO}

Para 1987 era notable el éxito de «algunos países del sudeste asiático» que habían seguido el modelo japonés que ha desarrollado «una nueva forma de incorporación al mundo del comercio que estimularía el progreso tecnológico» a través de «la creación deliberada de ventajas comparativas en los sectores que gozan de una demanda externa elástica» (Furtado, 1987: 224225). Ocurrió de esta manera gracias a una planificación para facilitar «un ordenamiento más integral que el proporcionado por los mercados» y a través de políticas industriales que se comprometiesen en «la necesaria reestructuración para superar el subdesarrollo» (Furtado, 1987: 225). Si bien Furtado arguyó acerca de una larga lista de fuerzas entrópicas que habían debilitado el potencial brasileño para sobrepasar las barreras estructurales y sumirse al prototipo «etapa avanzada del desarrollo», basó su explicación en la observación de casos probados de transformación socioeconómica y política que tuvieron lugar en otras naciones «seguidos de un largo periodo de inmovilidad» (Furtado, 1987: 227). 
Para alcanzar tal transformación, las naciones subdesarrolladas tendrían que emular la realidad que prevaleció durante el siglo XIX «cuando la ciencia se convirtió en el instrumento privilegiado de acumulación» (Furtado, 1983: 160). Para Furtado, quien impulsó el tema más que cualquier otra de las luminarias de la CEPAL, la tecnología era «la aplicación del conocimiento» y era el único medio con la capacidad de «estructurar el proceso de producción en términos de los criterios de la instrumentación racional», siendo el segundo «la esencia de la práctica social» necesaria para que una nación confronte las estructuras del subdesarrollo (Furtado, 1983: 165-167). Como se percibe fácilmente en los elementos presentados en la figura 1, en los dos modelos estructuralistas de Furtado la producción en cada sector requiere su propia gama de capacidades tecnológicas, con un ausencia de correspondencia entre los dos. Adicionalmente, las fuerzas endógenas del dinamismo surgido del cambio tecnológico - particularmente en una economía bíbrida- se encuentran totalmente ausentes o fuertemente truncadas. Desde esta perspectiva, se aprecia uno de los conceptos básicos del pensamiento de Furtado: la característica definitoria de una nación subdesarrollada, en relación con las llamadas naciones «desarrolladas», es su «heterogeneidad tecnológica» (Boianovsky, 2008:5).

\section{ESTRUCTURALISMO FURTADIANO VS. INSTITUCIONALISMO ${ }^{5}$}

La idea de que existen acentuadas similitudes y profundos puntos de convergencia entre el enfoque cepalista estructuralista y el institucionalismo estadounidense de Veblen y sus seguidores, ha sido ampliamente discutida, particularmente por James Street y Osvaldo Sunkel (Street, 1967, 1987; Sunkel, 1990). Extrañamente, Furtado rechazó categóricamente esta línea

\footnotetext{
${ }^{5} \mathrm{La}$ escuela institucionalista estadounidense fue bien establecida en la primera década del siglo $\mathrm{xx}$, si no antes (Hodgson, 2001). No hay ni la mínima correspondencia entre el llamado «nuevo institucionalismo» neoclásico que surgió en un intento de reformular el pensamiento neoclásico (véase Dugger, 1990).
} 
de análisis bajo la sospecha de que mientras él había publicado un libro diseñado para examinar la serie de ideas económicas que «consiguieron "explicar" el crecimiento económico»; «[...] los institucionalistas americanos fueron excluidos debido a que no presentaron una interpretación sistemática del proceso de crecimiento económico» (Furtado, 1964: viii, cursivas añadidas); por otro lado, y de manera sorprendente, Furtado no tenía consideraciones tan severas para el pensamiento neoclásico de Solow ${ }^{6}$ (Boianovsky, 2008: 21-23), incluso cuando un crítico calificado justificadamente rechazó el famoso modelo de «crecimiento» de Solow:

Una expansión sostenida se presenta en el modelo neoclásico sólo si se asume que existe un incremento autónomo de suministro de factores, consistentes en mano de obra y cambios autónomos en tecnología; ambos cambios son datos que no se explican. En este sentido, si se presenta una expansión sostenida, ésta ocurre por razones que no pueden encontrarse dentro de la teoría, por lo tanto, puede decirse que no hay tal teoría del crecimiento (Harris, 1975: 330, cursivas añadidas).

En contraste, Furtado aseveró:

El proceso de desarrollo involucra tanto nuevas combinaciones de factores existentes de un nivel técnico determinado o la introducción de innovaciones tecnológicas [...][;] podríamos tal vez considerar en un momento dado que son plenamente desarrolladas aquellas regiones en las que, en condiciones de pleno empleo de los factores, es posible aumentar la productividad únicamente mediante la introducción de innovaciones técnicas. Esta visión no es irreal [...] (Furtado, 1964: 61).

Aquí Furtado parece estar resumiendo el modelo de Solow, sin hacer referencia a él. No ofreció crítica alguna. Sin embargo, el modelo de Solow, así como también los modelos neoclásicos de crecimien-

${ }^{6}$ Con más de 18,000 citas académicas hasta la fecha, la obra magistral de Robert Solow, intitulada «A Contribution to the Theory of Economic Growth», fue publicada originalmente en 1956 por The Quarterly Journal of Economics vol. 70, núm. 1, pp. 65-94. 
to en general, se basan en supuestos fantasiosos (pleno empleo, equilibrio sostenido, competencia perfecta, rendimientos constantes de escala, etcétera); supuestos que «funcionan» sólo en la medida en que los componentes críticos de un sistema económico existente son descartados. En cualquier caso, el modelo de Solow, en las naciones avanzadas, puede explicar menos de la mitad del proceso de crecimiento en términos de factores (capital y mano de obra), y (heroica pero erróneamente) deja la explicación de la otra mitad del crecimiento a: 1) el cambio tecnológico (el cual en la teoría no es definido), o 2) la «ignorancia». Resulta notablemente contradictorio que Furtado, mientras concede aparente aceptación al modelo neoclásico de crecimiento, desprecia el institucionalismo estadounidense.

Por su parte, los institucionalistas se apresuraron a señalar y rechazar el argumento de Furtado (Junker, 1967). Junker destacó, en el primer número de la primera revista «heterodoxa» de la posguerra aparecida en Estados Unidos, Journal of Economic Issues, que Joan Robinson (a quien Furtado conoció durante su estancia en Cambridge en 1957/1958) encontraba al institucionalismo estadounidense muy prometedor para responder a las cuestiones planteadas por Furtado (Robinson, 1962: 105110). Descartando la teoría ortodoxa respecto a la cuestión crucial, «‡qué rige la tasa de acumulación de capital?», la economista Robinson resaltó: «Hay una teoría menos conocida que parece más prometedora, presentada por un discípulo de Veblen, el profesor C. E. Ayres» (Junker, 1967: 25-26). Ayres había escrito un importante trabajo, The Theory of Economic Progress (1944) (La teoría del progreso económico), el cual siguió una línea de análisis cuyo precursor fue Veblen. Enfatizó los papeles cruciales de las instituciones (factores retardadores) y la tecnología como los determinantes del proceso de crecimiento económico. Una lectura cuidadosa de Veblen demuestra su anticipada y premonitoria formulación de una serie de cuestiones relevantes tanto para el crecimiento económico como para el desarrollo económico (Cypher, 2009, 2012). Recayó entonces sobre Ayres, Junker, Street y otros la tarea de continuar con algunos de los originales aportes y prometedoras hipótesis anticipadas por Veblen. 
En el mismo número primero de la revista del institucionalismo, Street publicó «Los "estructuralistas" latinoamericanos e institucionalistas: convergencia en la teoría del desarrollo» (Street, 1967). En este artículo demostró que los estructuralistas habían sido rechazados por la corriente predominante de pensamiento económico por tener la insolencia de disentir de los postulados de la teoría económica neoclásica. Street encontró mucho mérito en una serie de trabajos estructuralistas, en particular citando a Pinto, Prebisch y Sunkel, y tomando muy poco en cuenta a Furtado. Irónicamente, y a la par de muchos elogios, Street notó que la mayor debilidad del estructuralismo latinoamericano era que éste «[... ] parecía haber tomado conciencia muy recientemente de la potencial relevancia del cambio tecnológico en el proceso doméstico de desarrollo» porque Prebisch y otros habían asumido que «la innovación tecnológica es algo disponible exclusivamente para el centro» (Street, 1967: 56). En general, la observación de Street acerca de que Latinoamérica en el siglo xix había caído bajo el control de «una clase social que no comprendía la cultura basada en la tecnología» era correcta y fue planteada con una presciencia perdurable (Street, 1987: 57). Como ha sido señalado en la sección precedente, Furtado fue un cepalista excepcional al haber señalado la importancia y el peso de la elusión de la tecnología y la ciencia como legado cultural disfuncional de la «economía colonial», legado que perduró en Brasil hasta la segunda mitad del siglo xix, y hasta el siglo xx en otros lugares (Furtado, 1971: 41; 1964: 133).

Los institucionalistas que han evolucionado enfrentan pocas dificultades para transferir la terminología «estructuralista» de los economistas de la CEPAL. "Las "estructuras" son, de hecho, "instituciones" » pero la «literatura estructuralista revela una asombrosa inocencia de su relación con los escritos institucionalistas norteamericanos» (Street, 1967: 55). En cuanto a la cuestión crucial de la tecnología, si bien Furtado la consideraba como el factor determinante en el proceso para salir del subdesarrollo, Street (basando su argumento en Ayres) ofreció una interpretación de este factor mucho más matizada y sofisticada, pues 
apreció su naturaleza, papel y ubicuidad en el marco de «una cultura basada en la tecnología »:

El término «tecnología» es tan amplio que actualmente parece una abstracción y, por lo tanto, una misteriosa fuerza disponible sólo para los iniciados. Esta concepción ha dado a menudo a los latinoamericanos una sensación de inferioridad desesperanzada frente al abrumador dominio tecnológico de los países más avanzados.

La tecnología, tomada como un todo, es una manera distintiva de pensar y de actuar e incluye nuevas formas de organización social y habilidades de gestión en las que el énfasis se encuentra en la eficiencia operativa. La incorporación de la tecnología en la cultura de los países menos desarrollados debe, por lo tanto, proceder en varios niveles más o menos de manera simultánea (Street, $1967: 59)$.

Los institucionalistas continuamente argumentaban que las estructuras institucionales represivas y anticuadas eran muy posiblemente los factores causales primarios de la perpetuación de las condiciones de subdesarrollo, incluso cuando haya sido el sistema colonial el que originalmente formó esas estructuras institucionales. De esta manera, en el análisis de los obstáculos al desarrollo, los factores internos se pondrían en el mismo plano que los factores externos (Street $1987: 1881-1882$ ). Este argumento se olvidó en la celeridad del análisis de la dependencia de mediados de la década de 1960 en adelante, sin embargo para Furtado no fue así, probablemente por su preferencia metodológica de un análisis históricamente contextualizado de la economía política.

Sin ninguna referencia específica a las publicaciones de Furtado, Sunkel —un economista legendario de la CEPAL - reconoció abiertamente que había sido un «intercambio desigual entre los institucionalistas y los estructuralistas» y que los estructuralistas «no habían hecho su tarea en relación con el institucionalismo» aun cuando en 1952 un argentino (Santiago Macario), quien otrora fuera estudiante de Ayres, publicó «una excelente introducción al institucionalismo» y posterior- 
mente trabajó en la CEPAL (Sunkel, 1990: 29). Sunkel argumentó entonces que por haber sido el estructuralismo cuestionado en América Latina tanto por neoclásicos como por marxistas «durante mucho tiempo pareció no haber ninguna necesidad de aliados», un comentario que no tiene ningún sentido (Sunkel, 1990:32).

\section{OBSERVACIONES FINALES}

La siguiente cita, sobre el trabajo de Prebisch, podría aplicarse igualmente, sino más, a la obra de Furtado:

Prebisch, durante sus primeros años, era bastante franco respecto a su admiración de la economía neoclásica. Mientras él sostenía que tenía la capacidad de reformular sus posturas, es debatible en la medida en que [...] se distanció él mismo del análisis neoclásico. En los temas que eligió analizar, asimismo la forma en que él y muchos de sus colegas [de la CEPAL] llevaron a cabo su análisis, y en la amplia gama de cuestiones y elementos que [la CEPAL] trató como exógenos a su análisis, hay más que un simple soplo de neoclasicismo (Cypher, 1990: 43-44).

La ambivalencia, o receptividad acrítica, de Furtado hacia la teoría económica ortodoxa fue un grave indicador de su incapacidad para comprender la naturaleza totalitaria de la ortodoxia y su predisposición a dejar en el exilio el pensamiento crítico. Es decir, una vez que la «economía» fue redefinida exitosamente por Robbins, esta transformación del campo dejó afuera cualquier posibilidad de que el análisis «histórico-estructural» fuera concebido como «economía» (Hodgson, 2001). A comienzos de la década de 1970, si no antes, era claro que una de las metas centrales de esta facción hegemónica era marginalizar, tanto como fuera posible, a cualquier economista profesional disidente (Mirowski, 2013: 177). 
Vale la pena recordar que Furtado argumentó que el análisis de las condiciones estructurales del subdesarrollo requería una ciencia económica nueva y distinta; que la economía ortodoxa no tenía la experiencia ni la capacidad de analizar las estructuras del subdesarrollo: «La economía del desarrollo, en su forma general, no entra en las categorías de análisis económico» (Furtado, 1958:316). No obstante, Furtado con frecuencia buscó una fusión parcial con la teoría neoclásica. Esto no ocurrió cuando él estaba inmerso en el análisis históricamente contextualizado de la estructura económica y su evolución en América Latina. Sin embargo, Furtado repetidamente ofreció disquisiciones sobre los aspectos del crecimiento y la economía del desarrollo, aquí recurriendo a una forma de presentación que fusionó el análisis neoclásico con el propio de Furtado (e.g., Furtado, 1964: 61-62, 64-65, 68, 77; Furtado, 1970:91-105).

Por otra parte, Furtado criticó específicamente el modelo de crecimiento de Kaldor, así también los modelos ofrecidos por Harrod y Domar, pues estos modelos derivaron de una «limitada experiencia histórica» (Furtado, 1964: 116). Por encima de su titubeante crítica, estos modelos no ofrecieron ninguna teoría del desarrollo. Y no podían ofrecerla dado que los supuestos se basaban en una economía homogénea, totalmente articulada e hipermoderna que opera en condiciones de pleno empleo, con sistemas financieros impecables e impulsada por grandes empresarios emprendedores (en lugar de una estructura de subdesarrollo interpenetrada por formas heterogéneas de producción operadas por legiones de rentistas oportunistas o por empresas familiares de muchas generaciones).

Furtado estaba bastante impresionado con la «función de progreso técnico» de Kaldor, la cual (en su construcción original) sostenía que la tasa de incremento en la productividad del trabajo era en función de la intensidad del capital por trabajador. ${ }^{7}$ Furtado citó de manera aprobatoria las formulaciones de Kaldor en al menos cuatro de sus últimos

${ }^{7}$ Véase, por ejemplo, el artículo clásico de Nicholas Kaldor y James Mirrlees de 1962: «A New Model of Economic Growth», The Review of Economic Studies, vol. 29, núm. 3, pp. 174-192. 
libros (Boianovsky, 2008: 22). Kaldor planteó la hipótesis de que la maquinaria nueva (adquirida con nueva inversión surgida, a su vez, del grado de intensidad del capital) trae consigo «cambio técnico incorporado», por lo tanto, a mayor grado de intensidad de capital, mayor grado de productividad como resultado del impacto de la nueva tecnología. El cómo el sistema de ecuaciones de Kaldor confrontaría el subdesarrollo, nunca fue aclarado. Una respuesta, sugerida por Sweezy en 1956, fue que el modelo no tiene que ver con el subdesarrollo: «[Kaldor] piensa que [ha presentado] el esquema de toda una teoría del desarrollo económico. Lo que realmente me asombra es la "propensión" de estos [los matemáticos, la construcción de modelos, los teóricos del crecimiento] a juguetear con unas pocas fórmulas - que son en el fondo meras tautologías - estableciendo conclusiones grandilocuentes a partir de ellas»(Baran, 2014: 38).

Sorprendentemente, quizás, Furtado tenía poco gusto por la hipótesis de Prebisch-Singer del deterioro secular de los términos de intercambio para los países productores de materias primas. Hasta antes de la Gran Depresión, la elasticidad del ingreso de la demanda de café permaneció alta y los términos de intercambio (1870-1929) mejoraron para Brasil (Bértola y Ocampo, 2012: 93; Boianovsky, 2008: 13-20). Brasil era conocido entonces como la «República del Café» (Font, 2010: 2). Furtado alegó queः «Si los países subdesarrollados tienen la oportunidad de invertir mirando al mercado exterior, no babría ningún problema. La cuestión fundamental, por lo tanto, es la ausencia de un mercado externo en expansión» (Furtado, 1958: 312, cursivas añadidas). En esta cita, Furtado no sólo impugnó la hipótesis más conocida de la CEPAL, sino también (de no matizar su argumento) transformó completamente la historia en su cabeza, omitiendo, por ejemplo, que México de 18701929 mostró un incremento de casi 10 veces en el volumen de exportaciones a la par de una profundización del subdesarrollo (incluyendo una disminución de 25 por ciento en los términos de intercambio), circunstancia que incubó, simultáneamente, las condiciones socioeconómicas para una revolución popular (Bértola y Ocampo, 2012: 93). Su comentario hace eco de lo peor de la «economía colonial», evocando la teoría 
del crecimiento del «desahogo del excedente» (vent for surplus) de Myint (y Smith) (Myint, 1958). Aparentemente, el argumento del pesimismo de exportación - el reconocimiento de que, en conjunto, los países subdesarrollados podrían (y lo hicieron en varias ocasiones) producir más productos primarios de los que el mercado mundial pudiera absorberhubiera sido lo suficientemente convincente (y suficientemente obvio) para que Furtado diera la espalda a las simplicidades reduccionistas de Myint/Smith. El hecho de que no lo hicieron aquí ni en otros trabajos, demuestra una inquietante predilección de Furtado para fusionar el análisis neoclásico con el suyo propio, argumentando al mismo tiempo, que sus objetivos requerían una teoría nueva e independiente.

La perturbadora pretensión de Furtado de fusionar teorías opues tas enfrentó un ambiente de creciente hostilidad ante su intento de construir una teoría fuera de las «categorías de análisis económico». Sin embargo, Furtado parecía no ser consciente del ataque neoliberal (sumamente agresivo) lanzado contra el desarrollo como un campo de análisis independiente; particularmente notable fue lo de P. T. Bauer a comienzos de la década de 1950; una línea consolidada más tarde por otros ideólogos de la Mont Pèlerin Society, como Anne Krueger y Deepak Lal (Mirowksi, 2013: 63; Plehwe, 2009). La falta de perspectiva de Furtado sobre este punto quizá tuvo mucho que ver con la curiosa cordialidad con que se desarrolló el debate acerca de la política de desarrollo en Brasil en 1950 (Sikkind, 1991: 66-67).

No obstante, Furtado representó la esencia misma del arquetipo del «intelectual orgánico» de Gramsci: comprometido, creativo, disciplinado, articulado, estratégico y erudito. Su liderazgo tuvo resultados perdurables en el tiempo en términos de la creación de un proyecto factible de desarrollo nacional. Esto fue particularmente ostensible durante las presidencias de Kubitschek (1956-1961), Quadros (1961) y Goulart (1961-1964), periodos en los que, como asesor presidencial clave, Furtado ocupó una serie de cargos de alto rango en el gobierno, contribuyendo a la construcción de la política de desarrollo. Furtado pudo renovar este papel a través de su influencia sobre los hacedores de políticas 
de desarrollo, quienes fueron instruidos e inspirados por el análisis furtadiano durante las administraciones neodesarrollistas de Luiz Inácio Lula da Silva (2003-2010) y el primer gobierno de Dilma Rousseff (2011-2014).

\section{BIBLIOGRAFÍA}

Alavi, Hamza (1982), «State and Class under Peripheral Capitalism», en Hamza Alavi y Theodor Shanin (eds.), Sociology of 'Developing Societies', Nueva York, Monthly Review Press, pp. 289-307.

Ayres, Clarence (1944), The Theory of Economic Progress, Chapel Hill, NC, University of North Carolina Press.

BAer, Werner (2001), The Brazilian Economy, Westport, CT, Praeger.

Baran, Nicholas (2014), "The Baran-Sweezy Letters Project», Monthly Review, vol. 65, núm. 10, pp. 30-48.

Bértola, Luis y José Ocampo (2012), The Economic Development of Latin America Since Independence, Oxford, Oxford University Press.

Blankenburg, Stephane, Gabriel Palma y Fiona Tregenna (2008), «Structuralism», en Steven Durlauf y Lawrence Blume (eds.), The New Palgrave Dictionary of Economics Online, 2da. ed., Houndmills, England, Palgrave Macmillan, pp. 1-9.

Boianovsky, Mauro (2008), «A View from the Tropics: Celso Furtado and the Theory of Economic Development in the 1950s», Textos para Discussão, TD 247, Brasil (outubro), Universidade Federal Fluminense, pp. 1-55.

(2014), «Between Lévi-Strauss and Braudel: Furtado and the HistoricalStructural Method in Latin American Political Economy», (febrero), pp. 1-37, en http: / / ssrn.com/abstract $=2392867$

Bresser-Pereira, Luiz (2007), «Method and Passion in Celso Furtado», en Esteban Pérez y Matías Vernengo (eds.), Ideas, Policies and Economic Development in the Americas, Londres, Routledge, pp. 9-30.

(2011), «An Account of New Developmentalism and its Structuralist Macroeconomics», Revista de Economía Política, vol. 31, núm. 3, pp. 493-502.

Cardoso, Fernando H. (2005), «Portrait of a Public Intellectual», Focus (abril), Brasilia, UNDP International Poverty Centre (IPC), pp. 3-5.

Corsi, Francisco y José Camargo (orgs.) (2010), Celso Furtado: os desafios do desenvolvimento, São Paulo, Editora Cultura Acadêmica. 
Cypher, James (1979), «The Internationalization of Capital and the Transformation of Social Formations», Review of Radical Political Economics, vol. 11, núm. 4, pp. 33-49.

(1990), «Latin American Structuralist Economics: An Evaluation, Critique and Reformulation», en James Dietz y Dilmus James (eds.), Progress Towards Development in Latin America: From Prebisch to Technological Autonomy, Boulder, CO, Lynne Rienner, pp. 41-65.

(2009), «On the Income Gap Between Nations: Was Veblen the First Development Economist?», Journal of Economic Issues, vol. 42, núm. 2, pp. 361-370.

(2012), «Veblen y el origen de la hipótesis del Catching-Up», Revista Problemas del Desarrollo, vol. 43 (abril-junio), pp. 9-26.

De Araúgo, Tarcicio, Júnior Macambira y Salvador Teixeira (orgs.) (2009), 50 anos de Formação Econômica do Brasil, Río de Janeiro, Instituto de Pesquicia Econômica Aplicada.

Dugger, William (1990), «The New Institutionalism: New but Not Institutionalist», Journal of Economic Issues, vol. 24, núm. 2, pp. 423-431.

Eatwell, John, Murray Millgate y Peter Newman (1989), The New Palgrave: Economic Development, Nueva York, W.W. Norton.

Font, Mauricio (2010), Coffee and Transformation in São Paulo, Brazil. Lanham, MD, Lexington Books.

Furtado, Celso (1958), «Capital Formation and Economic Development», en Amar Agarwala y Sampat Singh (eds.), The Economics of Underdevelopment, Nueva York, Oxford University Press, pp. 309-340.

(1963), «Brazil: What kind of Revolution?», Foreign Affairs, vol. 41, núm. 3, pp. 526-535.

(1964), Development and Underdevelopment, Berkeley, CA, University of California Press.

(1970), Obstacles to Development, Nueva York, Doubleday \& Company. (1971), The Economic Growth of Brazil: A Survey from Colonial to Modern Times, Berkeley, CA, University of California Press.

(1983), Accumulation and Development, Nueva York, St. Martin's Press. (1987), «Underdevelopment: to Conform or Reform», en Gerald Meier (ed.), Pioneers in Development, Second Series, Oxford, Oxford University Press, pp. 203-227.

Guillén, Arturo y Gregorio Vidal (2008), Celso Furtado, Madrid, Agencia Española de Cooperación Internacional para el Desarrollo. 
Harris, Donald (1975), «The Theory of Economic Growth: A Critique and Reformulation», American Economic Review, vol. 65, núm. 2, pp. 329-337.

Heilbroner, Robert y William Milberg (1995), The Crisis of Vision in Modern Economic Thought, Cambridge, Cambridge University Press.

Herrera, Rémy (2006), «The 'New’ Development Economics», Monthly Review, vol. 58, núm. 1, pp. 38-50.

Hodgson, Geoffrey (2001), How Economics Forgot History, Londres, Routledge.

Jomo K. S.y Erik Reinart (2005), The Origins of Development Economics, Londres, Zed.

Junker, Louis (1967), «Capital Accumulation, Savings-Centered Theory and Economic Development», Journal of Economic Issues, vol. 1, núm.1-2, pp. 25-43.

Krugman, Paul (1995), «The Fall and Rise of Development Economics», en Paul Krugman (ed.), Development, Geography and Economic Theory, Cambridge, мiт Press, pp. 1-30.

Mallorquin, Carlos (2005), Celso Furtado: um retrato intelectual, Río de Janeiro, Contraponto.

Miroswri, Phillip (2013), Never Let a Serious Crisis go to Waste, Londres, Verso.

Myint, Hla (1958), «The 'classical theory' of International Trade and the Underdeveloped Countries», Economic Journal, 68, pp. 317-337.

Palma, Gabriel (1978), «Dependency: a Formal Theory of Underdevelopment, or a Methodology for the Analysis of Concrete Situations of Underdevelopment?», World Development, 6, núm.7-8, pp. 881-924.

Plenwe, Dieter (2009), «The Origins of Neoliberal Economic Development Discourse», en Phillip Mirowski y Dieter Plehwe (eds.), The Road to Mont Pèlerin, Cambridge, Harvard University Press, pp. 238-279.

Robinson, Joan (1962), Economic Philosophy, Londres, C.A. Watts.

Siknind, Kathryn (1991), Ideas and Institutions: Developmentalism in Brazil and Argentina, Ithaca, NY, Cornell University Press.

Simon, David (ed.) (2006), Fifty Key Thinkers on Development, Londres, Routledge.

Schumpeter, Joseph (1930), «Mitchell's Business Cycles», Quarterly Journal of Economics, vol. 45, núm. 1, pp. 150-172.

Sombart, Werner (1930), «Capitalism», en Edwin Seligman y Alvin Johnson (eds.), Encyclopedia of the Social Sciences, vol. 3, Nueva York, Macmillan, pp. 195-208.

Street, James (1967), «The Latin American 'Structuralists' and the Institutionalists: Convergence in Development Theory», Journal of Economic Issues, vol. 1, núm. 1-2, pp. 44-62. 
(1987), «The Institutionalist Theory of Economic Development», Journal of Economic Issues, vol. 21, núm. 4, pp. 1861-1889.

Sunkel, Osvaldo (1990), «Structuralism, Dependency and Institutionalism: An Exploration of Common Ground and Disparities», en James Dietz y Dilmus James (eds.), Progress Towards Development in Latin America: From Prebisch to Technological Autonomy, Boulder, CO, Lynne Rienner, pp. 29-40.

Tavares, María (org.) (2000), Celso Furtado e o Brasil, São Paulo, Editora Fundação Perseu Abramo.

Topık, Stephen (1987), A Presença do Estado na Economia Politica do Brasil de 1889 a 1930, Río de Janeiro, Editora Record. 
